\title{
PSA 50 Percent or Greater than Nadir
}

National Cancer Institute

\section{Source}

National Cancer Institute. PSA 50 Percent or Greater than Nadir. NCI Thesaurus. Code C153408.

A finding indicating that the blood concentration of prostate specific antigen in a subject's sample has increased by 50 percent or more over the lowest value recorded for a sample from that subject. 